\title{
Coexistence of Cesarean Scar Endometriosis and Intramuscular Endometriosis
}

\author{
Duygu Kara Bozkurt ${ }^{\mathrm{a}}$, Murat Bozkurt ${ }^{\mathrm{b}, \mathrm{d}}$, Levent Sahin ${ }^{\mathrm{c}}$
}

\begin{abstract}
Endometriosis is defined as presence of functional endometrial tissue outside the uterus. With decreasing order of frequency, ovaries, peritoneum, subperitoneal deep pelvic cavity, intestinal and urinary system are the most common areas where the disease occurs. Endometriotic foci may develop on the scar tissue after previous surgery or abdominal wall in addition to pelvic region. This is often observed after obstetric and gynecological operations, and it needs to be distinguished with many different pathologies. Therefore, radiological modalities like ultrasound (US) imaging and magnetic resonance imaging (MRI) provide important clues for diagnosis. As far as we know, the coexistence of two separate foci, which are in subcutaneous fatty issue and in rectus muscles, has not been specified in any case report related to the abdominal scar endometriosis. In this case report, we intended to show a rare phenomenon, which is accompaniment of scar tissue endometriosis and rectus abdominis muscle endometriosis, by radiological imaging methods.
\end{abstract}

Keywords: Scar endometriosis; Intramuscular endometriosis; MRI

\section{Introduction}

Endometriosis is defined as presence of ectopic endometrial tissue and stroma outside the uterus. It can cause pelvic pain and infertility, and it is observed about $10 \%$ in reproductive age women [1]. Therefore, accurate diagnosis is very important. Radiological modalities are useful tools for diagnosis.

\footnotetext{
Manuscript accepted for publication March 4, 2014

a Department of Radiology, Kafkas University Medical Faculty, Kars, Turkey

${ }^{\mathrm{b}}$ Department of Obstetric and Gynecology, Kafkas University Medical Faculty, Kars, Turkey

${ }^{\mathrm{c}}$ Park Hospital IVF Center, Malatya, Turkey

${ }^{\mathrm{d}}$ Corresponding author: Murat Bozkurt, Kafkas Universitesi Saglik

Arastirma ve Uygulama Hastanesi Kars, Turkey.

Email: jindrmb@yahoo.com
}

doi: http://dx.doi.org/10.14740/jmc1728w
However, definitive diagnosis can be made by histopathology. Although endometriosis could be seen in almost every organ, ovaries, peritoneum, subperitoneal deep pelvic cavities, intestinal and urinary systems are the most common places where the disease observed [2]. Occasionally, it can be observed on the scar tissue and in intramuscular area at the level of the abdominal wall after obstetrics and gynecological surgeries (cesarean section, hysterectomy, episiotomy, tubal ligation, and so on). There are several case reports and case series about scar endometriosis and abdominal wall endometriosis in the literature. As far as we know, there has been no data about coexistence of scar tissue endometriosis and the rectus abdominis muscle endometriosis in the literature. In this case report, we discussed the coexistence of these two rare cases and its ultrasound (US) and magnetic resonance imaging (MRI) findings.

\section{Case Report}

A 36-year-old patient admitted to our clinic with ongoing cyclic pelvic pain and a palpable mass in the anterior abdominal wall for a period of 7 months. Interestingly, the patient described that this anterior abdominal wall mass swells and gives pain in each menstrual cycle. From the medical history, it was learned that patient had cesarean section 2 years ago. On physical examination, approximately $1.5 \mathrm{~cm}$ in diameter, hard and painful mass was palpated at the level of caesarean section scar in the anterior abdominal wall. Vaginal examination was normal and transvaginal ultrasonographic (TVUS) examination (Siemens Healthcare USA, S2000 Ultrasound System) showed that uterus and ovaries were normal in size. Endometrioma and pelvic endometriosis were not observed. The superficial US showed relatively smooth contoured hypoechoic solid lesions. One of them was $15 \times 14 \mathrm{~mm}$ in size on the right side of the incision in the subcutaneous fatty tissue; the other one was $14 \times 12 \mathrm{~mm}$ in size in the left rectus abdominis muscle. A pelvic MRI with contrast (Siemens Healthcare USA, Magnetom Avanto 1.5 Tesla MRI) was performed in order to visualize the lesion characterization more clearly. Lesions, which were on the right side of the incision in the subcutaneous fatty tissue 

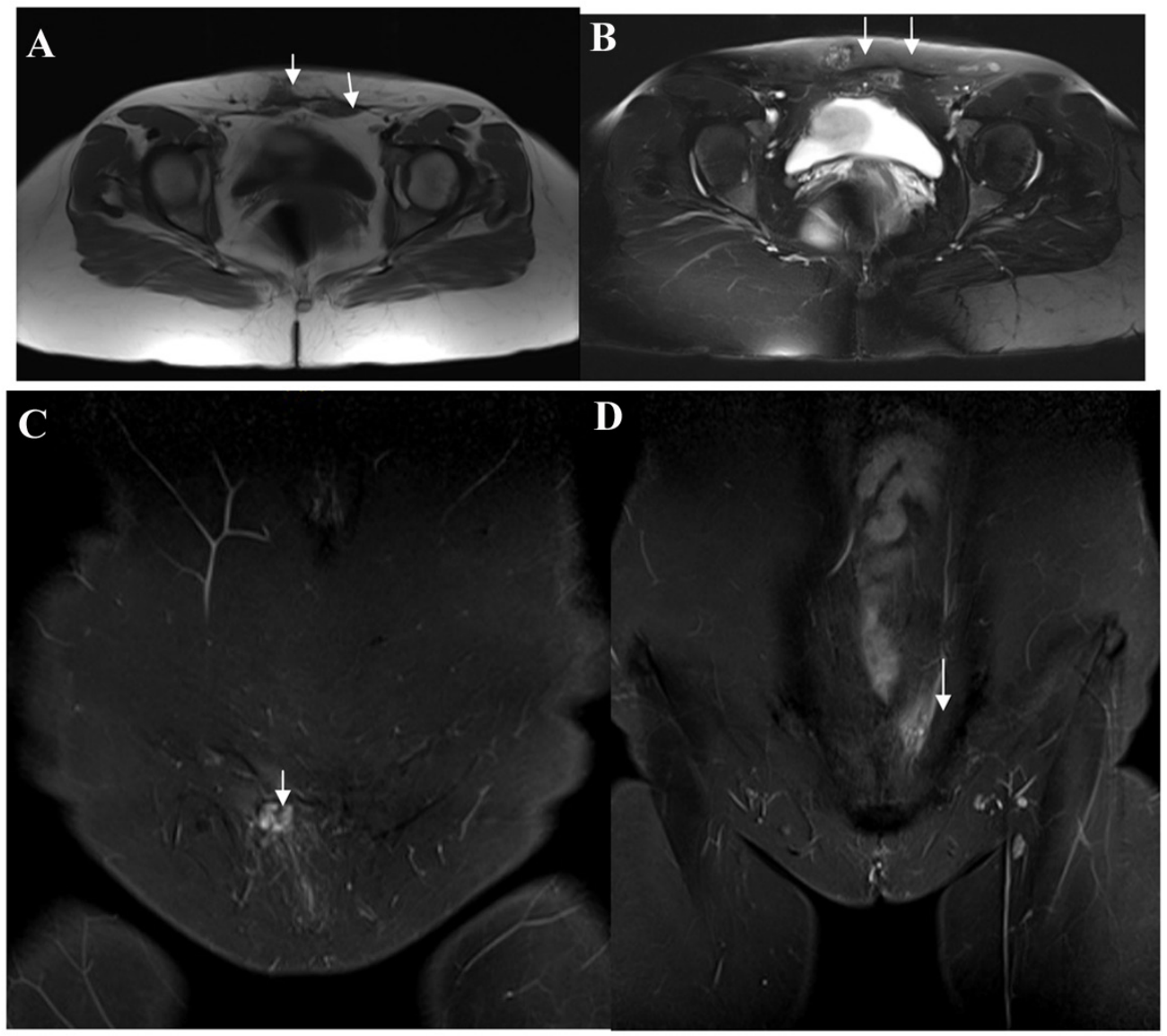

Figure 1. (A) Axial plan T1 TSE sequence; (B) Axial plan T2 FS TSE sequence; (C, D) Coronal plan T2 FS TSE sequences showing lesions, which were on the right side of the abdominal wall in the subcutaneous fatty tissue and in the left rectus abdominis muscle, were hypointense in coronal plan in T1A sequence, heterogeneous hyperintense in T2A and fat-suppressed T2-TSE sequence (white arrows).

and in the left rectus abdominis muscle, were hypointense in T1-TSE sequence, heterogeneous hyperintense in T2 TSE and fat-suppressed T2-TSE sequence (Fig. 1A-D) and they showed intense heterogeneous contrast enhancement in post-contrast T1-weighted images (Fig. 2A, B). Both lesions were hyperintense secondary to increased diffusion findings in diffusion-weighted imaging (DWI) and apparent diffusion coefficient (ADC) (Fig. 3A, B). At first, lesions were thought

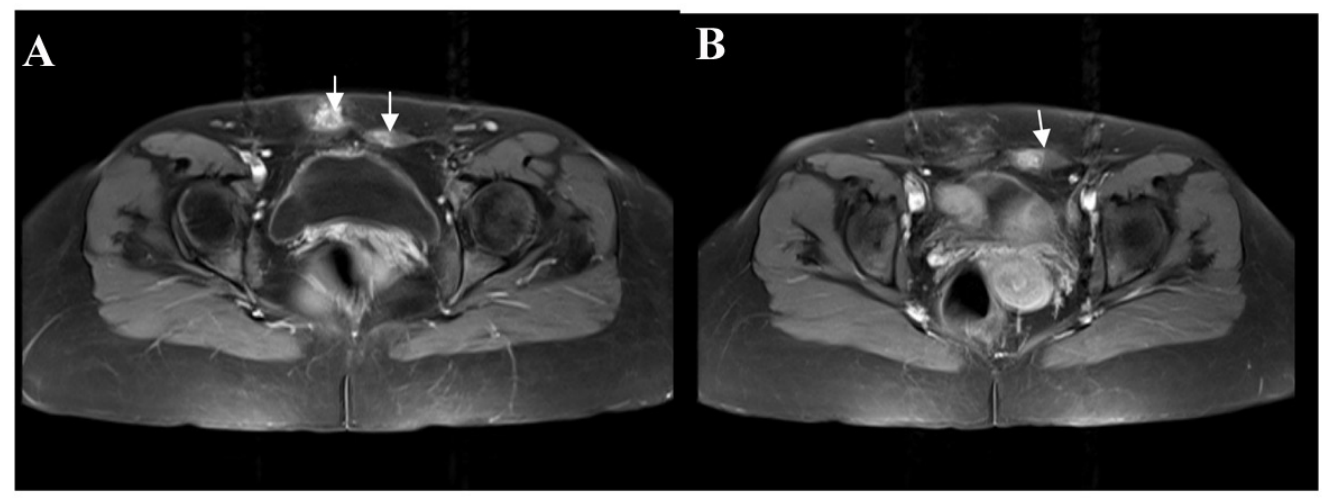

Figure 2. (A, B) Contrast enhanced T1-weighted fat suppressed sequences demonstrating heterogeneous enhancement patern in both lesions (white arrows). 


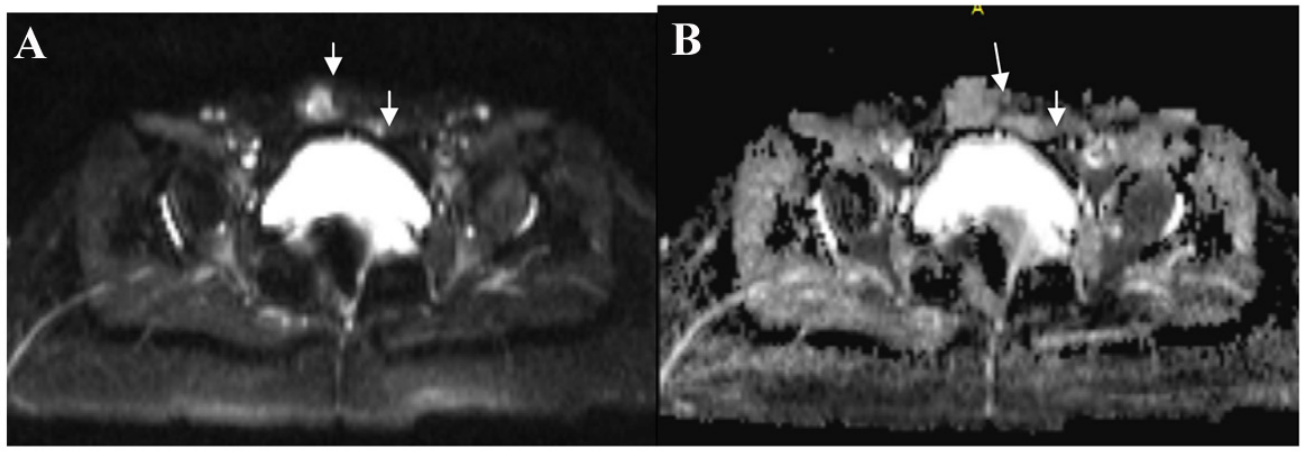

Figure 3. $(A, B)$ Both lesions were hyperintense secondary to increased diffusion findings in DWI and ADC (white arrows).

to be compatible with the endometriotic focus because of the patient's history of previous cesarean section, the presence of lesions with menstrual cycling pain and swelling, solid structure and intense contrast enhancement shown radiologically. Tumor excision was performed under general anesthesia. Pathology report showed that endometriosis foci in the subcutaneous fat tissue and muscle tissue with reactive lipogranulomatous inflammation.

\section{Discussion}

Endometriotic foci, which often develop at the level of incision and abdominal wall after gynecologic operations, are extremely rare. According to the literature the incidence of abdominal scar endometriosis after hysterectomy was 1.08$2 \%$, after cesarean section was $0.03-0.4 \%[3,4]$. Patients experience cyclic pain because of endometriotic foci which are hormone-dependent and bleed and growth in every menstrual cycle [5]. It is believed that scar endometriosis occurs as a result of iatrogenic implantation of endometriotic tissue secondary to gynecological surgery. A lot of case reports and case series in the literature show that the abdominal scar endometriosis can be seen on the skin, subcutaneous fatty tissue, the rectus abdominis sheath and in muscle [3, 6-8]. However, as far as we know, the coexistence of two separate foci, which are in subcutaneous fatty tissue and in rectus muscles, has not been specified in any case report related to the abdominal scar endometriosis. In this case report, without evidence of pelvic endometriosis, coexistence of two separate endometriotic foci, where in subcutaneous fatty tissue and rectus muscles, were evaluated with US and MRI methods. Endometriotic foci were observed in the solid appearance in US. MRI which has high soft tissue resolutions may be more appropriate in order to evaluate lesions more clearly because there are several situations in differential diagnosis such as hematoma, abscess, incisional hernia, suture granuloma, desmoid tumors, lymphoma and metastasis.

There are several advantages of MRI. First, it does not contain ionizing radiation. Second, it is a multiplanar imaging method. Third, it has high soft tissue resolution. All of these advantages are very useful both in diagnosis as well as preoperative localization and depth of the lesions, adjacent muscles plans and to reveal the relationship with intraabdominal organs and to detect intra-abdominal localization of different endometriotic foci. The only drawback of this method is the high cost. The presence of blood breakdown products in focal lesions in the anterior wall of the abdomen in MRI should suggest the diagnosis of endometriosis. However, definitive diagnosis can be made after excision and pathological evaluation of lesions. In some cases, fineneedle aspiration biopsy that is a minimally invasive method can be used for diagnosis and to rule out possible malignant condition. However, a major drawback of this technique is that pathological diagnosis cannot be made secondary to insufficient material obtainment in some cases.

In our case, the lesions are typically hypointense on T1weighted images, heterogeneous hyperintense secondary to the cystic-hemorrhagic foci on T2 TSE and fat-suppressed T2-weighted sequences. Post-contrast fat-suppressed T1weighted image shows the dense heterogeneous contrast enhancement. Both lesions were observed in an similar signal intensity. The age of blood breakdown products within the endometriotic focus affected lesion's signal intensity and $A D C$ values [9]. In our case, lesions were hyperintense in DWI and ADC. Therefore, increased diffusion sign suggests that the most lesions had cystic character. In one study, the $\mathrm{ADC}$ values were measured from the abdominal wall endometriosis and endometriotic foci. Endometriotic foci which show restricted diffusion signs, had high cellular density and contained very low amount of fibrotic tissue and smooth muscle hypertrophy [6].

Treatment should be determined according to the degree of the patient's symptoms. Medical therapy may be tried in patients with mild symptoms. Non-steroidal anti-inflammatory drugs, oral contraceptives, gonadotropin-releasing hormone analogues, aromatase inhibitors and radiofrequency ablation can be used for medical therapy [10]. Surgical 
therapy should be used in patients who do not respond to medical therapy. In order to prevent recurrence, wide total excision of the lesions until reaching negative surgical margins should be the method of choice as a surgical treatment. Pads used during surgery should not be left for a long time in the abdominal cavity, incision line should be washed and abdominal cavity closing should be done very carefully in patients with pelvic endometriosis in order to prevent scar endometriosis that might develop in the future [11]. Meshes may be used to reduce the likelihood of incisional hernia which might develop after a wide resection. In our case, the lesions were small for this reason incision line was closed primarily.

In the literature there are several case reports and case series regarding abdominal scar endometriosis. In our case, it is remarkable that there was an accompanying second focus in the rectus abdominis muscle which was different from the palpable focus on the scar tissue and this combination was revealed by MRI studies. As a result, normal physical examination and TVUS are insufficient to explain the etiology of pain in patients with chronic pelvic pain and had a history of surgery. In our opinion, assessment of patient with MRI evaluation would contribute significantly for the diagnosing the disease, detecting different endometriotic foci and increasing the success rate of treatment.

\section{References}

1. Siegelman ES, Oliver ER. MR imaging of endometriosis: ten imaging pearls. Radiographics. 2012;32(6):16751691.

2. Lahiri AK, Sharma K, Busiri N. Endometriosis of the uterine cesarean section scar: A case report. Indian J Radiol Imaging. 2008;18:1.

3. Kafkasli A, Franklin RR, Sauls D. Endometriosis in the uterine wall cesarean section scar. Gynecol Obstet Invest. 1996;42(3):211-213.

4. Goel P, Sood SS, Dalal A, Romilla. Cesarean scar endometriosis--report of two cases. Indian J Med Sci. 2005;59(11):495-498.

5. Kinkel K, Frei KA, Balleyguier C, Chapron C. Diagnosis of endometriosis with imaging: a review. Eur Radiol. 2006;16(2):285-298.

6. Busard MP, Mijatovic V, van Kuijk C, Hompes PG, van Waesberghe JH. Appearance of abdominal wall endometriosis on MR imaging. Eur Radiol. 2010;20(5):12671276.

7. Hensen JH, Van Breda Vriesman AC, Puylaert JB. Abdominal wall endometriosis: clinical presentation and imaging features with emphasis on sonography. AJR Am J Roentgenol. 2006;186(3):616-620.

8. Bennett GL, Slywotzky CM, Cantera M, Hecht EM. Unusual manifestations and complications of endometriosis-spectrum of imaging findings: pictorial review. AJR Am J Roentgenol. 2010;194:34-46.

9. Takahashi K, Okada S, Okada M, Kitao M, Kaji Y, Sugimura K. Magnetic resonance relaxation time in evaluating the cyst fluid characteristics of endometrioma. Hum Reprod. 1996;11(4):857-860.

10. Akbulut S, Sevinc MM, Bakir S, Cakabay B, Sezgin A. Scar endometriosis in the abdominal wall: a predictable condition for experienced surgeons. Acta Chir Belg. 2010;110(3):303-307.

11. Emre A, Akbulut S, Yilmaz M, Bozdag Z. Laparoscopic trocar port site endometriosis: a case report and brief literature review. Int Surg. 2012;97(2):135-139. 